\title{
Anti-CD122 Humanized Monoclonal Antibody Mik-Beta-1
}

National Cancer Institute

\section{Source}

National Cancer Institute. Anti-CD122 Humanized Monoclonal Antibody Mik-Beta-1. NCI

Thesaurus. Code C121231.

A humanized version of the immunoglobulin (Ig) G1 monoclonal antibody Mik-Beta-1

(Hu-Mik-Beta-1) directed against CD122, the beta-subunit shared by the interleukin-2 (IL2) and IL-15 receptor (IL-2/IL-15Rbeta). Upon intravenous infusion, Hu-Mik-Beta-1 binds to CD122 expressed on certain tumor cells. This blocks the binding of the inflammatory cytokines IL-2 and IL-15 to IL-2R and IL-15R, respectively, and prevents IL-2/IL-2R- and IL-15/IL-15R-mediated signaling. This may inhibit the proliferation of CD122-expressing tumor cells. In addition, blocking CD122 on T-lymphocytes prevents the over-activation of T-lymphocytes in various T-cell mediated autoimmune diseases, which is predominantly mediated by IL-15/IL-15R signaling. CD122, involved in both IL-2 and IL-15 signaling, is overexpressed on certain cancer cells, such as those found in T-cell large granular lymphocyte (T-LGL) leukemia. 